\title{
USING SURFACE NORMALS TO LOCALIZE SUBDURAL INTRACRANIAL ELECTRODES PLACED DURING NEUROSURGERY
}

\author{
James P. O'Shea, William M. Wells III, and Alexandra J. Golby \\ Departments of Neurosurgery and Radiology \\ Brigham and Women's Hospital and Harvard Medical School, Boston, MA, USA \\ $\{$ joshea $\mid$ sw $\mid$ agolby $\} @$ bwh.harvard.edu
}

\begin{abstract}
Clinical and research neurosurgical studies involving the use of intracranial electroencephalography and cortical stimulation depend critically on accurate localization of electrodes. Neuronavigation tracking probes enable surgeons to record the position of exposed electrodes, but they cannot be used to reliably measure subdural contacts placed under the dura and beyond the extent of the craniotomy. We describe an algorithm to estimate the position of these inaccessible sites using the patient's preoperative structural MRI data. After generating a model of the cortical surface, our algorithm estimates surface normals at the sites of accessible electrodes, establishes the orientation of the electrode strips, and then extrapolates the position of adjacent contacts based on the known inter-electrode distance. We performed validation using a patient dataset consisting of 37 electrodes placed on the cortical surface during surgery.
\end{abstract}

\section{INTRODUCTION}

Numerous studies have demonstrated the clinical benefits of intracranial electroencephalography (iEEG) and direct electrocortical stimulation (DECS) during awake neurosurgical procedures [1, 2]. In addition to their clinical use, these techniques provide a unique opportunity to study human brain function. Research in this field depends critically on accurate measurements of the intracranial electrode positions as a means to both effectively interpret the accompanying data, and to properly correlate the results with other imaging modalities such as fMRI. Previous studies have approximated these positions by taking intraoperative photographs of the cortical surface and matching vascular and sulcal anatomy to define the location [3]. The growing use of neuronavigational

The authors gratefully acknowledge support from NIH (AJ Golby: K08NS048063, U41-RR019703; WM Wells: P41-RR13218, R01-LM00786101A1, U54-EB005149), The Brain Science Foundation, and Brigham and Women's Institute for the Neurosciences. We acknowledge GE Healthcare Navigation (Lawrence, MA) for the development and technical support of Image Fusion and Surgical Navigation products. Dr. Golby presently has no financial relationship with GE Healthcare but has served as an unpaid consultant for the development of the GE Healthcare Navigation System.

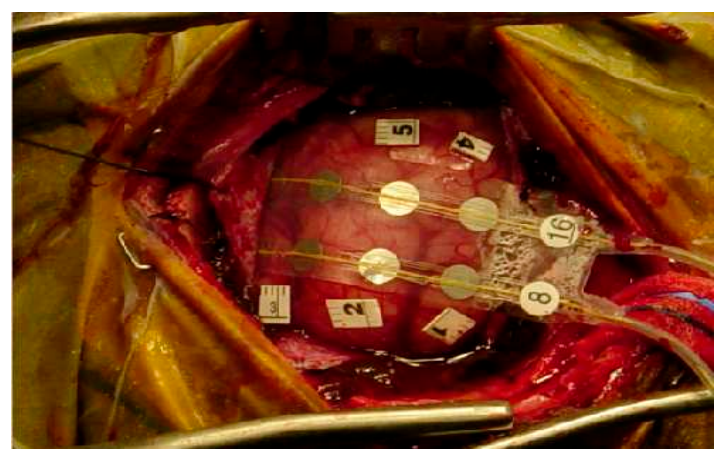

Fig. 1. Intraoperative photograph of the surgical site shows the small craniotomy and cortical surface. A $2 \times 8$ electrode grid has been placed sliding the distal 8 contacts under the edge of the craniotomy. The location of those electrodes can only be roughly estimated by placing the neuronavigation probe on the scalp surface. Note: the validation data used in this paper were collected during a different case.

computer systems during surgery provides the opportunity to decrease the error in electrode localization, and more recent studies have demonstrated qualitative and quantitative comparisons within the neuronavigation systems [4].

The need to establish physical contact between the system's tracking probe and the relevant locations, however, presents a considerable limitation in accurately localizing subdural electrodes positioned beyond the extent of the craniotomy (Fig. 1). This is particularly relevant since the widespread adoption of intraoperative neuronavigation has allowed surgeons to approach tumors and lesions through smaller craniotomies. To help remedy this situation, the surgeon may visually approximate the location of these sites by registering a point on the scalp directly above the electrode (Fig. 2). This measurement method can easily lead to spurious results however, adversely affecting any subsequent data analyses that are contingent on accurate electrode positions. In cases in which electrodes are placed on the inferior temporal lobe in particular, the surgeon is often unable to provide even a gross estimation of their lo- 

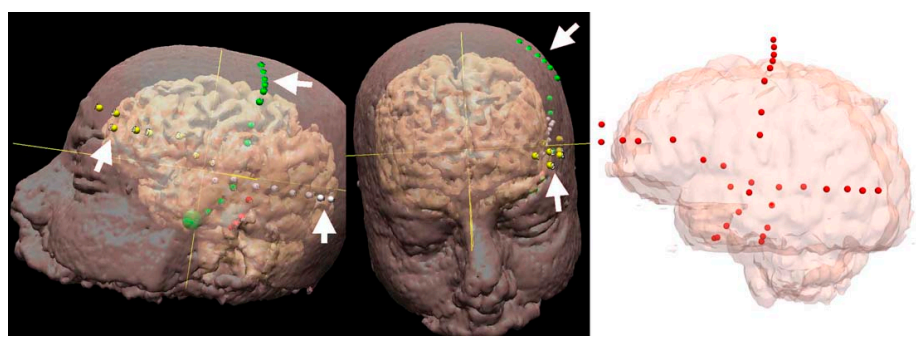

Fig. 2. Left,Center: Intraoperative screenshots from the neuronavigation system showing the recorded electrode locations. The surgeon visually approximated on the scalp the positions of any electrodes inaccessibly placed under the dura and beyond the reach of the tracking probe (white arrows). Right: Postoperative integration of these data in the 3D Slicer[5] application for input to the algorithm. We generated the model of the cortical surface from the preoperative MRI data, and the electrode positions (red markers) from the neuronavigation coordinates.

cations due to the intervening bone and muscle tissue. The algorithm we present automatically extrapolates the positions of these inaccessible electrodes based solely on the known characteristics of the electrode strip and the cortical surface model generated from the preoperative imaging data.

\section{SURGICAL PROCEDURE}

Surgical navigation was conducted using the InstaTrak frameless stereotactic system (GE Healthcare Navigation, Lawrence, MA). The reference MRI volume was a high-resolution T1weighted gradient echo 3D MPRAGE (Magnetization Prepared Rapid Gradient Echo) image $(256 \times 256$ matrix; $240 \mathrm{~mm}$ FOV; 124 slices). The surgeon applied the electrode strips to the cortical surface according to the clinical needs of the patient, and the positions of accessible electrodes were registered on the neuronavigation system using the hand-held tracking probe. When possible, inaccessible electrodes placed beyond the extent of the craniotomy (i.e. beyond the reach of the tracking probe) were approximately localized by recording a point on the scalp visually estimated to be directly above the electrode's intracranial location (Fig. 2). A CT scan of the patient was collected with the implanted electrodes in place to provide validation data. The intracranial electrodes used during the craniotomy were composed of platinum contacts embedded in a flat strip of flexible plastic (AD-TECH Medical Instrument Corporation, Racine WI). Each strip consisted of 8 contacts spaced $10 \mathrm{~mm}$ apart in a $1 \times 8$ configuration. We assume the strips provide enough flexibility to conform to the curved surface of the cortex with minimal stretching or lateral bending. The validation data presented below demonstrate that this is a reasonable assumption.

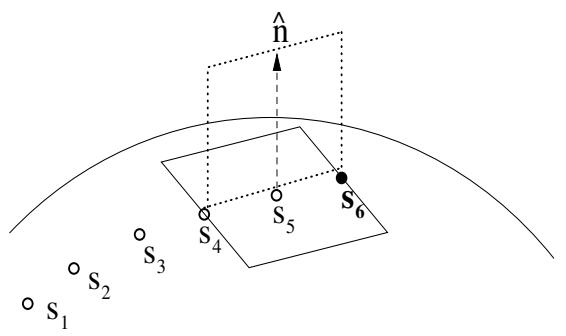

Fig. 3. Diagram illustrating the algorithm steps to estimate the next contact site $\left(s_{6}\right)$ on an electrode strip. We estimate the normal ( $\hat{\mathbf{n}})$ to the cortical surface at the most distal site $\left(s_{5}\right)$, and then establish the orientation of the strip with the plane that is parallel to $\hat{\mathbf{n}}$ and passes through $s_{4}$ and $s_{5}$. We select the solution $\left(s_{6}\right)$ by minimizing 1$)$ the distance to this plane, and 2) the inter-electrode spacing error.

\section{POSTOPERATIVE DATA PROCESSING}

We used the 3D Slicer[5] software to integrate the preoperative MRI volume with the electrode sites registered by the neuronavigation system. After segmenting the cortex from the reference MRI volume, we generated a model $R$ of point cloud data (PCD) in $\mathbb{R}^{3}$ which approximately represented this surface. Electrode positions recorded by the neuronavigation system were converted to the proper coordinate space and rendered alongside the cortical model (Fig. 2).

Our algorithm estimates adjacent subdural electrode positions given: 1) the locations of other contacts from the same strip and, 2) an accurate model of the cortical surface. The first input is the initial set $S$ of $n$ contact sites which were accessible to the tracking probe and accurately recorded. In order to establish the orientation of the electrode strip, $n \geq 2$. The model $R$ of the cortical surface is the second input parameter. The algorithm begins estimation at the first site beyond the craniotomy. Each newly estimated site is added to $S$ before iterating for the next electrode localization.

\section{SURFACE NORMALS}

Given the set $S$ of accurate initial contact sites and the cortical model $R$, we establish the orientation of the electrode strip by calculating a surface normal $\hat{\mathbf{n}}$ at the contact site $s_{n}$ closest to the edge of the craniotomy. The surface normal is estimated by solving the well-known least-squares method to find the direction of minimum dispersion for the cloud of nearby model points[6]. Given a point cloud $X$ composed of the $k$ nearest neighbors to $s_{n}$ (represented by column vectors), we find the plane which passes through the center of mass of $X$ and minimizes the sum of the squared distances from the plane to each of the points in $X$. Let $\bar{x}=\frac{1}{k} \sum_{i=1}^{k} x_{i}$ be the center of mass 
for $X$. If the plane through $\bar{x}$ is defined by its unit normal $\mathbf{n}$, then the distance from $x_{i}$ to the plane is $d_{i}=\mathbf{n}^{T}\left(x_{i}-\bar{x}\right)$. We want to minimize $E(\mathbf{n})=\sum_{i=1}^{k} d_{i}{ }^{2}$. Thus $E(\mathbf{n})=\mathbf{n}^{T} M \mathbf{n}$ where

$$
M=\sum_{i=1}^{k}\left(x_{i}-\bar{x}\right)\left(x_{i}-\bar{x}\right)^{T}
$$

Give that $\mathbf{n}$ is a unit vector and therefore $\mathbf{n}^{T} \mathbf{n}=1$, we introduce a Lagrange multiplier $\lambda$ and then differentiate:

$$
\begin{aligned}
E(\mathbf{n}) & =\mathbf{n}^{T} M \mathbf{n}-\lambda \mathbf{n}^{T} \mathbf{n} \\
\frac{d E}{d \mathbf{n}} & =2 M \mathbf{n}-2 \lambda \mathbf{n}
\end{aligned}
$$

This results in the eigenvalue problem: $M \mathbf{n}=\lambda \mathbf{n}$. If $\mathbf{n}_{i}$ is a normalized eigenvector representing a possible solution to this problem, then we minimize:

$$
E\left(\mathbf{n}_{i}\right)=\mathbf{n}_{i}^{T} M \mathbf{n}_{i}=\mathbf{n}_{i}^{T} \lambda_{i} \mathbf{n}_{i}=\lambda_{i}
$$

Note $\lambda_{i} \geq 0$. Therefore $\mathbf{n}_{i}$ must be the minimum eigenvalue, and we choose the estimated $\hat{\mathbf{n}}=\mathbf{n}_{i}$ as the minimum unit eigenvector that is normal to the least-squares plane.

\section{STRIP ORIENTATION}

Once the surface normal $\hat{\mathbf{n}}$ is estimated at the $s_{n}$ site, we extrapolate the position of the next contact in the strip by calculating the plane that is parallel to $\hat{\mathbf{n}}$ and passes through the two most distal contacts $s_{n}$ and $s_{n-1}$ from the set $S$ of initial sites. This plane effectively represents the orientation of the leading end of the electrode strip, and the position of the next electrode in the strip is found by minimizing the distance to this plane. The plane is defined by its unit normal $\hat{\mathbf{n}}_{p}$ as follows:

$$
\begin{aligned}
\mathbf{n}_{p} & \equiv\left(s_{n}-s_{n-1}\right) \times \hat{\mathbf{n}} \\
\hat{\mathbf{n}}_{p} & =\frac{\mathbf{n}_{\mathbf{p}}}{\left|\mathbf{n}_{\mathbf{p}}\right|}
\end{aligned}
$$

The placement of the next contact site in the strip is found by iterating through the remaining points in the model and minimizing: 1) the distance to this plane, and 2) the interelectrode spacing error. For any point $r_{j}$ in the model $R$, the distance to the plane is calculated as $\varepsilon_{1}=\left[\hat{\mathbf{n}}_{p}^{T}\left(r_{j}-s_{n}\right)\right]^{2}$. Let $\alpha$ be the known distance between contacts on the electrode strip. The measure of inter-electrode spacing error is therefore $\varepsilon_{2}=\left[\left|r_{j}-s_{n}\right|-\alpha\right]^{2}$. A solution is then found by iterating through the model points and minimizing:

$$
F=\varepsilon_{1}+\varepsilon_{2}=\left[\hat{\mathbf{n}}_{p}^{T}\left(r_{j}-s_{n}\right)\right]^{2}+\left[\left|r_{j}-s_{n}\right|-\alpha\right]^{2}
$$

The resulting point $r_{j} \in R$ is thus chosen as the most likely adjoining contact position on the electrode strip. If estimating additional sites, we simply add $r_{j}$ to $S$ and then iterate through the algorithm again. In this way, the results from one iteration are used as input to the next. The results from a single strip are depicted in Fig. 4. The algorithm is illustrated in Fig. 3 and summarized below.

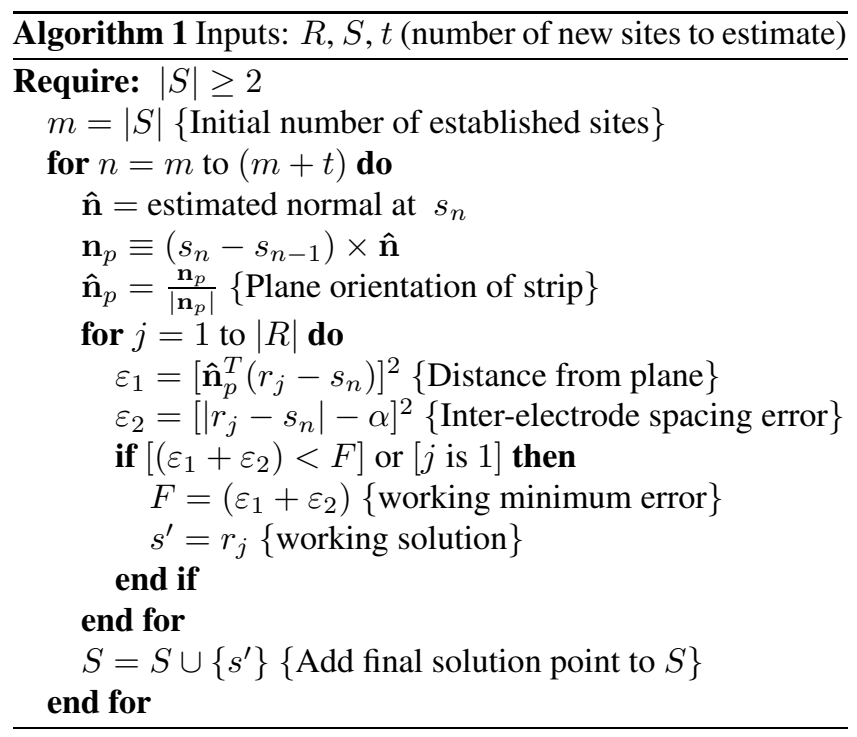

\section{EXPERIMENTAL RESULTS}

We validated the results of our algorithm using a patient dataset consisting of 5 electrode strips placed during surgery (four $1 \times 8$ strips, one $1 \times 5$ strip). Twenty of the 37 total electrodes employed in the procedure were located subdurally beyond the extent of the craniotomy. The surgeon approximated the position of 16 of these contacts on the patient's scalp. The remaining 4 contact sites were positioned on the inferior temporal lobe, where the intervening bone and tissue prevented any scalp approximations to be made. We determined the actual electrode positions using a postoperative CT volume.

The algorithm produced a mean error of $7.7 \pm 0.7 \mathrm{~mm}$ for all 20 inaccessible sites. Comparing only the 16 sites which were also approximated by the surgeon, the algorithm resulted in in a mean error of $8.0 \pm 1.8 \mathrm{~mm}$ compared to 23.9 $\pm 0.9 \mathrm{~mm}(p<0.001)$ using the intraoperative approximations. The algorithm estimated the positions of the 4 electrodes placed on the inferior temporal lobe within $6.4 \pm 1.8$ $\mathrm{mm}$ of the actual positions.

\section{DISCUSSION}

The method we describe allows precise localization of electrodes which are positioned beyond the extent of the craniotomy and cannot be accurately measured by the neuronavigation system. Neurosurgical advances, particularly the use of intraoperative neuronavigation, have resulted in the ability to perform significantly smaller craniotomies, thus decreasing patient morbidity and healing time[7]. However, in many 

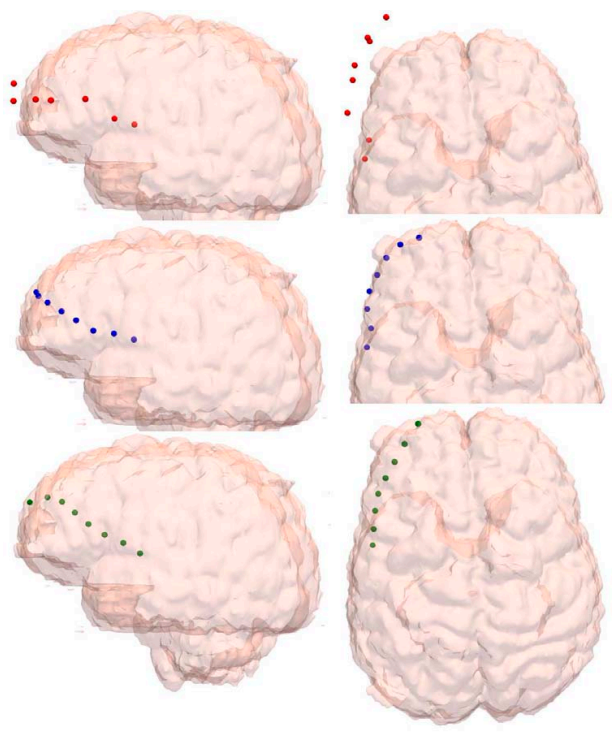

Fig. 4. Left and superior views of the algorithm input, output, and validation data for one strip. Top (red): Intraoperative locations recorded by neuronavigation system. The surgeon approximated the six anterior sites on the scalp because they were inaccessible to the tracking probe. Middle (blue): Algorithm results for the same strip. All contacts are now flush with the surface. Bottom (green): Validation data from CT.

cortical mapping cases, the surgeon must make a larger craniotomy in order to test the relevant brain functions. The ability to place electrode strips under the dura and skull beyond the extent of the craniotomy allows the testing of a much larger area of cortex while still preserving the advantages of a smaller craniotomy. A further benefit of our algorithm reveals itself when electrodes are placed on the inferior temporal lobe, where intervening bone and tissue structure prevent even a gross estimation of the electrode positions using the tracking probe. Our algorithm provides the only option for accurately localizing contact sites in this area.

The utility of our method is primarily relevant to intracranial EEG data acquisition conducted during surgery, since most postoperative EEG monitoring can utilize CT imaging to localize implanted electrodes. For this reason, our method of validation may lead to problems in error assessment. Although we can reliably determine electrode positions using the CT scan, these data may not accurately reflect their true intraoperative positions. Electrodes can shift when the surgeon closes the dura and replaces the bone, and brain shift may occur as a result of edema, physiologic respiratory changes, or fluid changes. Despite these shortcomings, this validation method remains the best option that is readily available.

Another potential source of error concerns the quality of the cortical surface PCD. The algorithm finds a solution se- lected from this set of points, and an inaccurate or an insufficiently dense PCD could lead to poor results. Since solutions from one iteration of the algorithm may serve as input to the next, any errors can potentially propagate through to subsequent sites on a given strip. The fact that our data do not exhibit this trend is most likely because the brain shift that occurred prior to the CT scanning displaced the electrodes within the vicinity of the craniotomy, pushing them below the level of the preoperative cortical surface. The error actually decreased with each iteration of the algorithm since the CT data converged with the cortical surface as the distance from the craniotomy increased. (see Fig. 4, lower-right).

Despite these limitations, research involving iEEG and DECS can significantly benefit from the algorithm we present. Although more work validating the results is needed, this method represents a significant improvement in localizing intracranial electrodes when the neuronavigation system cannot be used.

\section{REFERENCES}

[1] G.A. Ojemann, J. Ojemann, E. Lettich, and M.S. Berger, "Cortical language localization in left, dominant hemisphere: an electrical stimulation mapping investigation in 117 patients," $J$. Neurosurgery, vol. 71, pp. 316-326, 1989.

[2] J.Ph. Lachaux, D. Rudrauf, and P. Kahane, "Intracranial eeg and human brain mapping," J. Physiology Paris, vol. 97, pp. 613-628, 2003.

[3] J. Hirsch, M. Ruge, K. Kim, D. Correa, J. Victor, N. Relkin, D. Labar, G. Krol, M. Bilsky, M. Souweidane, L. DeAngelis, and P. Gutin, "An integrated functional magnetic resonance imaging procedure for preoperative mapping of cortical areas associated with tactile, motor, language, and visual functions," Neurosurgery, vol. 47, pp. 711-721, 2000.

[4] F.E. Roux, K. Boulanoauar, J.A. Lotterie, M. Mejdoubi, J.P. LeSage, and I. Berry, "Language functional magnetic resonance imaging in preoperative assessment of language areas: correlation with direct cortical stimulation," Neurosurgery, vol. 52, pp. 1335-1345, 2003.

[5] D.T. Gering, A. Nabavi, R. Kikinis, W.E.L. Grimson, N. Hata, P. Everett, F.A. Jolesz, and W.M. Wells III, "An integrated visualization system for surgical planning and guidance using image fusion and interventional imaging.," in MICCAI, 1999, pp. 809819.

[6] M.J. Mitra, A. Nguyen, and L. Guibas, "Estimating surface normals in noisy point cloud data," International Journal of Computational Geometry and Applications, vol. 14, pp. 261276, 2004.

[7] D.L. Hill, A.D. Smith, A. Simmons, C.R. Maurer Jr., T.C. Cox, R. Elwes, M. Brammer, D.J. Hawkes, and C.E. Polkey, "Sources of error in comparing functional magnetic resonance imaging and invasive electrophysiological recordings," J. Neurosurg, vol. 93, pp. 214-223, 2000. 